\title{
Mathematical Procedures for the Non-Coplanar Tangential Transfers between Circular Orbits
}

\author{
A. A. Alqarni \\ Department of Mathematics, Faculty of Science, University of Jeddah, Jeddah, KSA \\ Email: Alyah141414@gmail.com
}

How to cite this paper: Alqarni, A.A. (2021) Mathematical Procedures for the Non-Coplanar Tangential Transfers between Circular Orbits. Open Access Library Journal, 8: e7101.

https://doi.org/10.4236/oalib.1107101

Received: December 22, 2020

Accepted: January 19, 2021

Published: January 22, 2021

Copyright $\odot 2021$ by author(s) and Open Access Library Inc.

This work is licensed under the Creative Commons Attribution International License (CC BY 4.0).

http://creativecommons.org/licenses/by/4.0/ (c) (i) Open Access

\begin{abstract}
To transfer a satellite or a spacecraft from a low parking orbit to another orbit requires one of the many orbital transfers. These orbital transfers need to determine some orbital elements of the initial and final orbits as perigee and apogee distances. The transfers compete to achieve the transition with minimal consumption of energy, transfer time, as well as the highest accuracy of transition. In the present research, certain mathematical procedures implementable with the help of computers will be employed to investigate the two important non-coplanar tangential transfers between circular orbits called the Hohmann and bi-elliptic transfers. Also, a comparative study between Hohmann and bi-elliptic transfers will be established. At the end of present study, we will be able to determine the lowest value of the velocity change $\Delta v$ and the best transfer between the Hohmann and bi-elliptic transfers with minimal fuel consumption.
\end{abstract}

\section{Subject Areas}

Applied Statistical Mathematics, Classical Mechanics

\section{Keywords}

Orbital Transfer, Hohmann Transfer, Bi-Elliptic Transfer, Non-Coplanar Maneuver

\section{Introduction}

The subject of mathematics has been found to play vital roles in many science and technological professions including the space science and astronomy to state a few. The present space technological advancements resulted in maneuvering or rather transferring satellites and spacecrafts from low parking orbits to the final desired orbits. These transfers battle to attain minimal time, consumption of 
energy and the highest accuracy of transition among others. In particular, a satellite accomplishes orbital maneuvers to rectify the shape, size, or the site of a satellite [1]. In some stages for most satellites, we need to change the orbital elements of a satellite. The orbital elements that need to be changed depend on whether the orbits are in the same plane or non-coplanar. When a spacecraft is launched to the space or to the orbit around the Earth, is it placed in the beginning at the initial orbit or law parking orbit, and then to appropriate orbit. Transferring a spacecraft from one orbit to the other requires a large amount of fuel [2]. The spaceship is transferred to a final orbit in which it is planned to spend its life or accomplish its mission by using different types of transfer orbits. The transfer orbit can be defined in orbital mechanics as an intermediate elliptical orbit that is used to move a spacecraft from one orbit to the other. There are several types of transfer orbits that vary in their required energy efficiency and speed of transfer [3] [4]. For more relevant literature on the orbital maneuvering for spacecraft and rockets, one can see [5] [6] [7] [8] [9] and the references therewith. However, in the present research, certain mathematical procedures implementable with the help of computers will be employed to investigate the two important non-coplanar tangential transfers between circular orbits called the Hohmann and bi-elliptic transfers. Also, we are conducting a comparative study of these conversions. At the end of the present study, we can determine which among the transfers has the lowest value of the velocity change $\Delta v$ with minimal fuel consumption. The organization of the paper takes the form: Section 2 gives the complete analysis of the Hohmann transfer; while Section 3 gives that of bi-elliptic transfer. Section 4 presents the computational algorithms of the transfers. Section 5 discusses the obtained results, and Section 6 gives some concluding remarks.

\section{The Hohmann Transfer}

In this section, we give a complete analysis of the Hohmann orbital transfer comprising of transfers on the same plate and plane change, and the determination of maximum value and plane changes among others.

\subsection{Hohmann Transfer on the Same Plane}

The Hohmann orbital transfer is one of the most famous transfers. It moves between two circular or elliptical orbits of dissimilar radii in the same plane with the lowest possible amount of energy and highest fuel efficiency. Figure 1 shows the velocity of the first impulse, $v_{a}$, and the velocity of the second impulse, $v_{b}$, can be determined from vis-viva equation and by using the velocities of the initial (LER) and final (GEO) orbits, $v_{c_{1}}$ and $v_{c_{2}}$ given respectively by

$$
\begin{aligned}
& v_{c_{1}}=\sqrt{\frac{\mu}{r_{1}}}, \\
& v_{c_{2}}=\sqrt{\frac{\mu}{r_{2}}},
\end{aligned}
$$




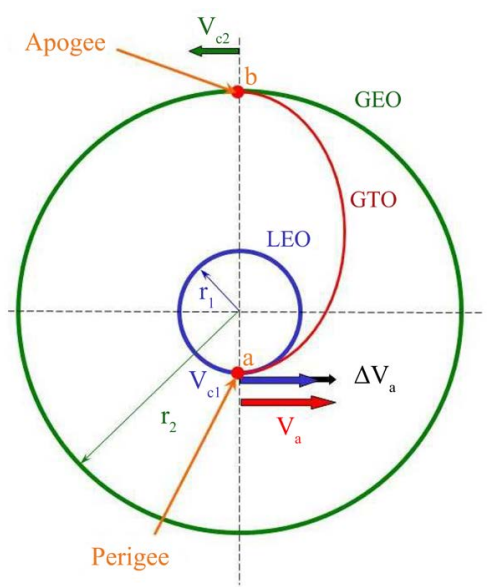

Figure 1. Hohmann transfer.

$$
\begin{aligned}
& v_{a}=\sqrt{\frac{2 \mu}{r_{1}}-\frac{\mu}{a}}, \\
& v_{b}=\sqrt{\frac{2 \mu}{r_{2}}-\frac{\mu}{a}} .
\end{aligned}
$$

The first impulse is given as:

$$
\Delta v_{a}=\left|v_{a}-v_{c_{1}}\right|=\sqrt{\frac{\mu}{r_{1}}}\left(\sqrt{\frac{2 r_{2}}{r_{1}+r_{2}}}-1\right) .
$$

The second impulse is given as:

$$
\Delta v_{b}=\left|v_{c_{2}}-v_{b}\right|=\sqrt{\frac{\mu}{r_{2}}}\left(1-\sqrt{\frac{2 r_{1}}{r_{1}+r_{2}}}\right) .
$$

The total impulse for the Hohmann transfer is:

$$
\Delta v_{H}=\Delta v_{a}+\Delta v_{b},
$$

or

$$
\frac{\Delta v_{H}}{v_{c_{1}}}=\left(1-\frac{1}{R}\right) \sqrt{\frac{2 R}{1+R}}+\sqrt{\frac{1}{R}}-1
$$

where $R=\frac{r_{2}}{r_{1}}, \quad a=\frac{r_{1}+r_{2}}{2}$ is the Semi-major axis of transfer ellipse, and $\mu=398600.44 \mathrm{~km}^{3} / \mathrm{s}^{2}$ represents the Earth's mass gravitational constant.

\subsection{The Maximum Value of Hohmann Transfer}

Differentiating Equation (8) w.r.t. $R$ and equating the resultant to zero, we obtain

$$
\frac{1}{R^{2}}\left(\frac{2 R}{1+R}\right)^{\frac{1}{2}}+\frac{R-1}{R(1+R)^{2}}\left(\frac{2 R}{1+R}\right)^{-\frac{1}{2}}-\frac{1}{2 R^{\frac{3}{2}}}=0
$$

Then it is easy to obtain the following from Equation (6) after multiplying by 
$\sqrt{2}(1+R)^{\frac{3}{2}} R^{\frac{3}{2}}$ and thereafter squaring both sides,

$$
F(R)=R^{3}-15 R^{2}-9 R-1=0 .
$$

Now, since the coefficients in Equation (10) have only one change in the sign, only one real root is posed based on the Descartes rule, and is given by

$$
R=R_{M}=5+4 \sqrt{7} \cos \left(\frac{1}{3} \tan ^{-1} \frac{\sqrt{3}}{37}\right)=15.58176 \cdots
$$

The Maximum value of $\frac{\Delta v_{H}}{v_{c_{1}}}$ is

$$
\left(\frac{\Delta v_{H}}{v_{c_{1}}}\right)_{\max }=0.536258
$$

It is remarkable to mention here that for the bi-elliptic transfer, the limiting value $R$ is determined to be $R=R_{M}=15.58176$, which is exactly the same as in the Hohmann transfer.

\subsection{Superposition of Escape and Return Trajectory and Hohmann Transfer}

The non-dimensional velocity increase for the total maneuver is

$$
\begin{gathered}
\Delta v_{\infty}=\Delta v_{\infty} e+\Delta v_{\infty} r, \\
\frac{\Delta v_{\infty}}{v_{c_{1}}}=(\sqrt{2}-1)\left(1+\frac{v_{c_{2}}}{v_{c_{1}}}\right) .
\end{gathered}
$$

Then, we get

$$
\frac{\Delta v_{\infty}}{v_{c_{1}}}=(\sqrt{2}-1)\left(1+\sqrt{\frac{1}{R}}\right) .
$$

As Figure 2 shows, the curves of $\Delta v_{H}$ and $\Delta v_{\infty}$ intersect. To seek for the point of intersection, it is possible to equate Equations (8) and (15)

$$
\left(1-\frac{1}{R}\right)\left(\frac{2 R}{1+R}\right)^{\frac{1}{2}}-1=(\sqrt{2}-1)\left(1+\left(\frac{1}{R}\right)^{\frac{1}{2}}\right)-\left(\frac{1}{R}\right)^{\frac{1}{2}} \text {. }
$$

multiply by $\sqrt{R}$ and squaring both sides of the resulting equation, we then get

$$
\frac{2(R-1)^{2}}{1+R}=2 R+2 \sqrt{2 R}(\sqrt{2}-2)+(\sqrt{2}-2)^{2},
$$

that is

$$
\frac{1-3 R}{R+1}-(3-2 \sqrt{2})=\sqrt{R}(2-2 \sqrt{2}),
$$

multiply by $R+1$ and squaring both sides of the resulting equation, we get

$$
\begin{aligned}
& (1-3 R)^{2}-2(1-3 R)(1+R)(3-2 \sqrt{2})+(1+R)^{2}(3-2 \sqrt{2})^{2} \\
& =(1+R)^{2} R(2-2 \sqrt{2})^{2}
\end{aligned}
$$




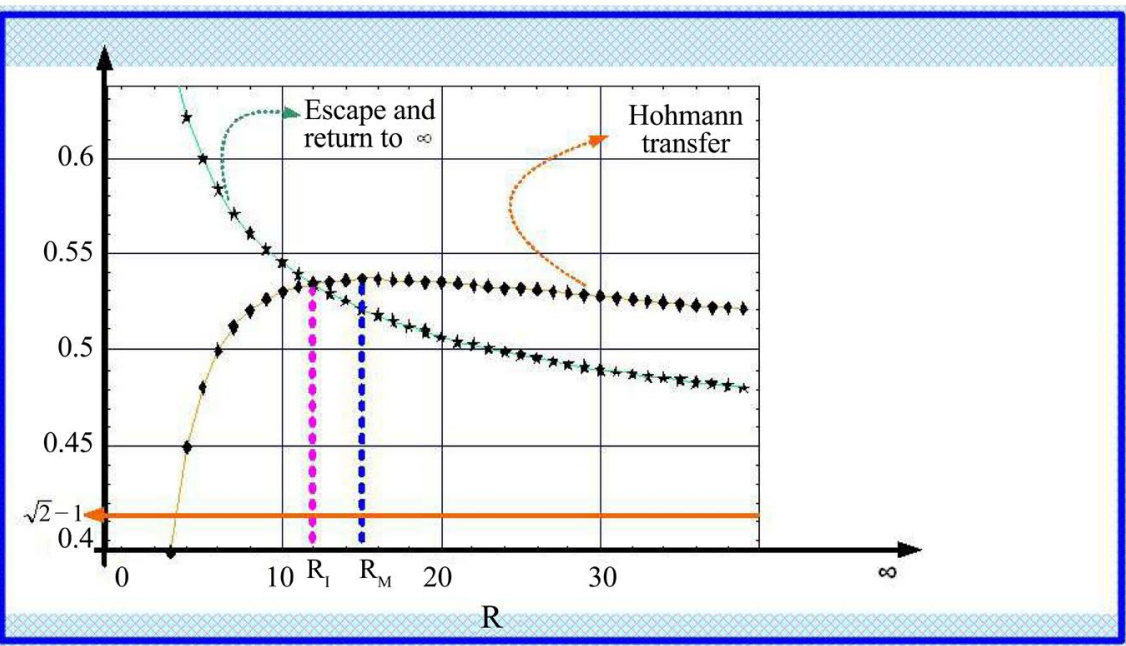

Figure 2. Superposition of escape and return trajectory and Hohmann transfer.

after some little analysis, we get

$$
1+\frac{4 R(7-6 \sqrt{2})}{4(3-2 \sqrt{2})}+\frac{4 R^{2}(5-2 \sqrt{2})}{4(3-2 \sqrt{2})}-R^{3}=0 .
$$

The required equation is thus obtained as

$$
R^{3}-(7+4 \sqrt{2}) R^{2}+(3+\sqrt{2}) R-1=0 .
$$

Now, since the coefficients in Equation (17) have only one change in the sign, then three roots are posed based on the Descartes rule, and given by

$$
R=0.146554, R=0.571535 \text {, and } R=11.93876 \text {. }
$$

We therefore reject the first two roots since we care about the case when $r_{2}>r_{1}$; i.e. $R>1$. So, the only root in this case is:

$$
R=R_{1}=11.9387655 .
$$

\subsection{Plane Change Transfers}

To derive the velocity increments at each plane change, consider the vector diagram in Figure 3. This vector diagram gives the geometric relationships between, for example the initial velocity $v_{x}$, the velocity on the transfer orbit $v_{y}$, and the total velocity change $\Delta v_{1}$. On using the law of cosines, we get:

$$
\Delta v_{j}=\sqrt{v_{x}^{2}+v_{y}^{2}-2 v_{x} v_{y} \cos \theta_{j}}, \quad j=1,2,3 .
$$

In the same way, we can find the second and third velocities $\Delta v_{2}$ and $\Delta v_{3}$. Therefore, the increment at all three plane changes is given by:

$$
\Delta v_{\text {total }}=\Delta v_{1}+\Delta v_{2}+\Delta v_{3} \text {. }
$$

\subsection{Hohmann Transfer with Plane Change}

\section{Transfer modes (Figure 4)}




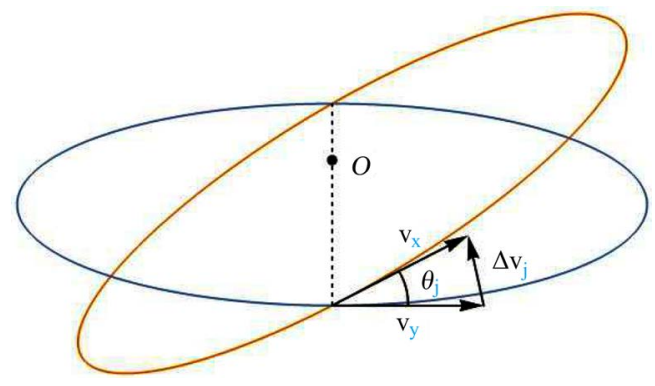

Figure 3. Velocity triangle.

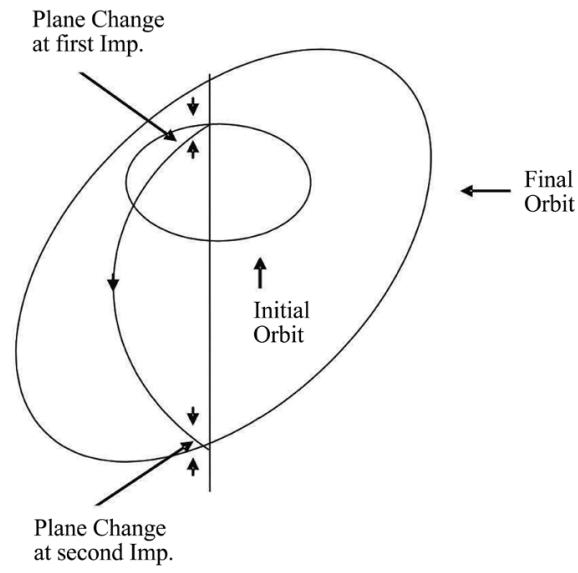

Figure 4. Hohmann transfer with plane change.

1) The Hohmann transfer is an optimal two impulse transfer. A departure is made from initial orbit with plane change at first impulse.

2) Plane change at second impulse results from ellipse orbit to final orbit. A circular consideration is also considered with regards to the initial and final orbits.

The first plane change

From Equations (1) and (3), by using Equation (19), $\theta=\theta_{1}$, we have:

$$
\frac{\Delta v_{a}}{v_{c_{1}}}=\left(1+Q_{1}^{2}-2 Q_{1} \cos \theta_{1}\right)^{\frac{1}{2}},
$$

where

$$
Q_{1}=\sqrt{\frac{2 r_{2}}{r_{1}+r_{2}}}=\sqrt{\frac{2 R}{1+R}}
$$

The second plane change

From Equations (2) and (4), by using Equation (19), $\theta=\theta_{2}$, we have:

$$
\frac{\Delta v_{b}}{v_{c_{1}}}=Q_{2}\left(1+Q_{3}^{2}-2 Q_{3} \cos \theta_{2}\right)^{\frac{1}{2}},
$$

where

$$
Q_{2}=\sqrt{\frac{r_{1}}{r_{2}}}=\sqrt{\frac{1}{R}} \text { and } Q_{3}=\sqrt{\frac{2 r_{1}}{r_{1}+r_{2}}}=\sqrt{\frac{2}{R+1}} \text {. }
$$


The total velocity increment of Hohmann transfer can be obtained from Equation (20) as:

$$
\frac{\Delta v_{H}}{v_{c_{1}}}=\left(1+Q_{1}^{2}-2 Q_{1} \cos \theta_{1}\right)^{\frac{1}{2}}+Q_{2}\left(1+Q_{3}^{2}-2 Q_{3} \cos \theta_{2}\right)^{\frac{1}{2}} .
$$

\section{The Bi-Elliptic Transfer}

In this section, we give a complete analysis of the bi-elliptic orbital transfer comprising of transfers on the same plate and plane change, and the determination of the plane changes among others.

\subsection{Bi-Elliptic Transfer on the Same Plane}

The bi-elliptic orbital transfer is the orbital maneuvering that transfers a spacecraft from one orbit to the other. The transfer mode of the bi-elliptic transfer is shown in Figure 5.

The bi-elliptic transfer's initial impulse tangentially exerted at the initial (first) circular orbit with radius $r_{1}$ is given as:

$$
\Delta v_{a}=\sqrt{\frac{2 \mu}{r_{1}}-\frac{\mu}{a_{1}}}-\sqrt{\frac{\mu}{r_{1}}},
$$

where $a_{1}=\frac{r_{1}+r_{i}}{2}$ denotes the Semi-major axis of first elliptic transfer orbit. By substituting $a_{1}$ into Equation(24), we get:

$$
\frac{\Delta v_{a}}{v_{c_{1}}}=\sqrt{\frac{2 r_{i}}{r_{1}+r_{i}}}-1
$$

The second impulse of the bi-elliptic transfer tangentially exerted at the apoapsis of the first elliptic transfer orbit is given as:

$$
\frac{\Delta v_{b}}{v_{c_{1}}}=\sqrt{\frac{2 r_{1}}{r_{i}}}\left(\sqrt{\frac{1}{1+\frac{r_{i}}{r_{2}}}}-\sqrt{r_{1}} \sqrt{\frac{1}{r_{1}+r_{i}}}\right) .
$$

The third impulse is given as:

$$
\frac{\Delta v_{c}}{v_{c_{1}}}=\sqrt{\frac{r_{1}}{r_{2}}}\left(\sqrt{\frac{2 r_{i}}{r_{2}+r_{i}}}-1\right) .
$$

The total impulse for the bi-elliptic transfer is thus determined by summing Equations (25), (26) and (27) as:

$$
\frac{\Delta v_{B}}{v_{c_{1}}}=\sqrt{\frac{2 S}{1+S}}-1+\sqrt{\frac{2}{S}}\left(\sqrt{\frac{1}{1+\frac{S}{R}}}-\sqrt{\frac{1}{1+S}}\right)+\sqrt{\frac{1}{R}}\left(\sqrt{\frac{2 S}{R+S}}-1\right),
$$

where $S=r_{i} / r_{1}$. 


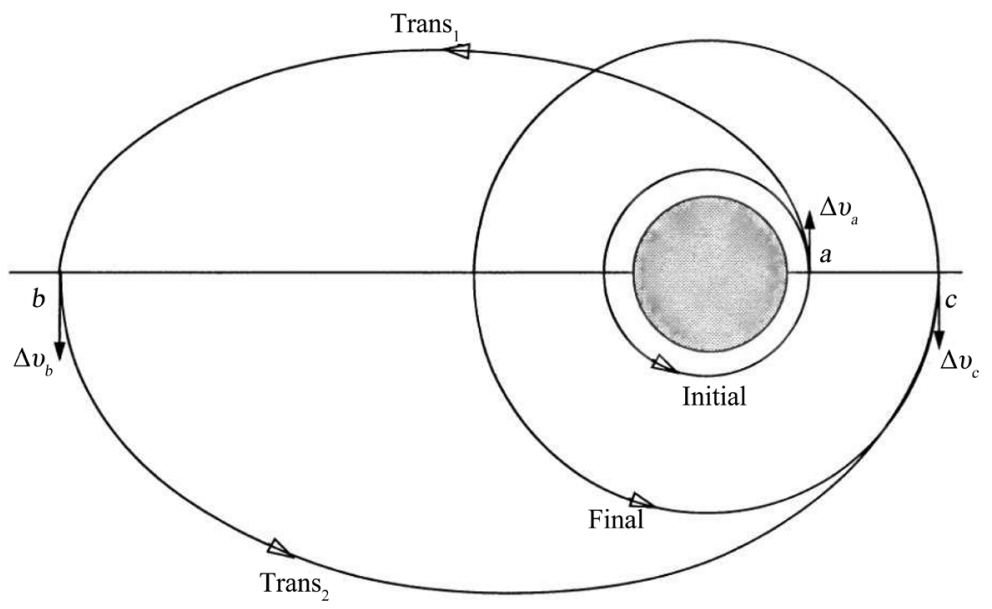

Figure 5. Bi-elliptic transfer.

\subsection{Bi-Elliptic Transfer with Plane Change}

\section{Transfer mode (Figure 6)}

1) A departure is made from orbital plane 1 with plane change angle $\theta_{1}$.

2) At apoapsis of the resulting elliptic orbit, the second impulse exerted to change the orbit plane through an angle $\theta_{2}$.

3) Finally, at apapsis of the resulting second ellipse a final plane change through an angle $\theta_{3}$ puts the vehicle in the desired final orbit, that is the orbital plane 2. The initial and final orbits are taken to be circular.

The first plane change

$$
\frac{\Delta v_{a}}{v_{c_{1}}}=\left(1+H_{1}^{2}-2 H_{1} \cos \theta_{1}\right)^{\frac{1}{2}},
$$

where

$$
H_{1}=\sqrt{\frac{2 r_{i}}{r_{1}+r_{i}}}=\sqrt{\frac{2 S}{1+S}} .
$$

\section{The second plane change}

$$
\frac{\Delta v_{b}}{v_{c_{1}}}=H_{2}\left(1+H_{3}^{2}-2 H_{3} \cos \theta_{2}\right)^{\frac{1}{2}}
$$

where

$$
H_{2}=\sqrt{\frac{2 r_{1} r_{2}}{r_{i}\left(r_{2}+r_{i}\right)}}=\sqrt{\frac{2 R}{S(R+S)}} \text { and } H_{3}=\sqrt{\frac{r_{1}\left(r_{2}+r_{i}\right)}{r_{2}\left(r_{1}+r_{i}\right)}}=\sqrt{\frac{R+S}{R(1+S)}} .
$$

The third plane change

$$
\frac{\Delta v_{c}}{v_{c_{1}}}=H_{4}\left(1+H_{5}^{2}-2 H_{5} \cos \theta_{3}\right)^{\frac{1}{2}},
$$

where

$$
H_{4}=\sqrt{\frac{r_{1}}{r_{2}}}=\sqrt{\frac{1}{R}} \text { and } H_{5}=\sqrt{\frac{2 r_{i}}{r_{2}+r_{i}}}=\sqrt{\frac{2 S}{R+S}} \text {. }
$$




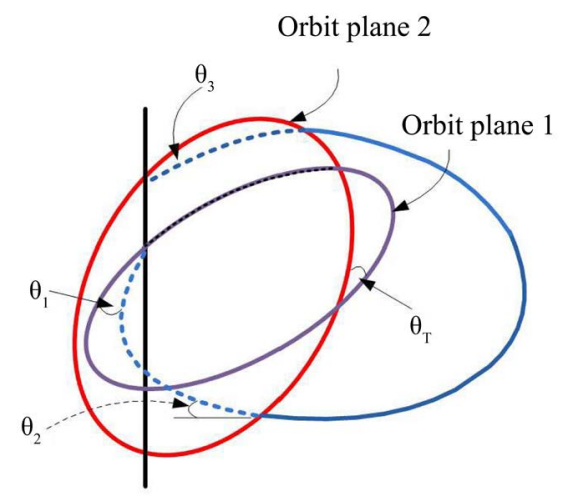

Figure 6. Bi-elliptic transfer with plane change.

By using Equation (20) and from Equations (29), (30) and (31), we have for the total velocity increment relative to the initial velocity $v_{c_{1}}$ the following expression:

$$
\begin{aligned}
\frac{\Delta v_{B}}{v_{c_{1}}}= & \left(1+H_{1}^{2}-2 H_{1} \cos \theta_{1}\right)^{\frac{1}{2}}+H_{2}\left(1+H_{3}^{2}-2 H_{3} \cos \theta_{2}\right)^{\frac{1}{2}} \\
& +H_{4}\left(1+H_{5}^{2}-2 H_{5} \cos \theta_{3}\right)^{\frac{1}{2}} .
\end{aligned}
$$

\section{Computational Development of Hohmann and Bi-Elliptic Transfers with Plane Change}

This section outlines the two algorithms designed for both the Hohmann and bi-elliptic transfers with the help of Mathematica software.

Module list for Hohmann transfer

$$
\begin{aligned}
& \operatorname{HOHM}\left(\mathrm{r} 1_{-}, \mathrm{r} 2_{-}, \theta 1_{-}, \theta 2_{-}\right):=\operatorname{Module}[\{m=1\}, \\
& \mathrm{vc} 1=\sqrt{\frac{m}{\mathrm{r} 1}} ; \mathrm{vc} 2=\sqrt{\frac{m}{\mathrm{r} 2}} ; \\
& \mathrm{dva}=\sqrt{\frac{m}{\mathrm{r} 1}} \sqrt{-2 \cos (\theta 1) \sqrt{\frac{2 \mathrm{r} 2}{\mathrm{r} 1+\mathrm{r} 2}}+\frac{2 \mathrm{r} 2}{\mathrm{r} 1+\mathrm{r} 2}+1 ;} \\
& \mathrm{dvb}=\sqrt{\frac{m}{\mathrm{r} 1}} \sqrt{\frac{\mathrm{r} 1}{\mathrm{r} 2}} \sqrt{-2 \cos (\theta 2) \sqrt{\frac{2 \mathrm{r} 1}{\mathrm{r} 1+\mathrm{r} 2}}+\frac{2 \mathrm{r} 1}{\mathrm{r} 1+\mathrm{r} 2}+1 ;} \\
& \mathrm{dvH}=\mathrm{dva}+\mathrm{dvb}] .
\end{aligned}
$$

\section{Module list for bi-elliptic transfer}

BIELLEPTIC(r1_,r2_,ri_, $\left.\theta 1_{-}, \theta 2_{-}, \theta 3_{-}\right):=$Module[ $\{m=398599$.$\} ,$

$$
\begin{aligned}
& \mathrm{dva}=\sqrt{\frac{m}{\mathrm{r} 1}} \sqrt{-2 \cos (\theta 1) \sqrt{\frac{2 \mathrm{ri}}{\mathrm{r} 1+\mathrm{ri}}+\frac{2 \mathrm{ri}}{\mathrm{r} 1+\mathrm{ri}}+1}} ; \\
& \mathrm{dvb}=\sqrt{\frac{m}{\mathrm{r} 1}} \sqrt{\frac{\mathrm{r} 1 \mathrm{r} 2}{\mathrm{ri}(\mathrm{r} 2+\mathrm{ri})}} \sqrt{-2 \cos (\theta 2) \sqrt{\frac{\mathrm{r} 1(\mathrm{r} 2+\mathrm{ri})}{\mathrm{r} 2(\mathrm{r} 1+\mathrm{ri})}}+\frac{\mathrm{r} 1(\mathrm{r} 2+\mathrm{ri})}{\mathrm{r} 2(\mathrm{r} 1+\mathrm{ri})}+1 ;} \\
& \mathrm{dvc}=\sqrt{\frac{m}{\mathrm{r} 1}} \sqrt{\frac{\mathrm{r} 1}{\mathrm{r} 2}} \sqrt{-2 \cos (\theta 3) \sqrt{\frac{2 \mathrm{ri}}{\mathrm{r} 2+\mathrm{ri}}}+\frac{2 \mathrm{ri}}{\mathrm{r} 2+\mathrm{ri}}+1} ; \\
& \mathrm{dvB}=\mathrm{dva}+\mathrm{dvb}+\mathrm{dvc}] .
\end{aligned}
$$




\section{Results and Discussion}

It is noted from the investigation carried out so far that there exist $R_{M}$ and $R_{I}$ as the two critical ratios. These ratios give the following four inequalities:

$$
1<R<9, \quad 9 \leq R \leq R_{I}, \quad R_{I}<R \leq R_{M}, \quad R>R_{M} .
$$

Thus, a comparison analysis between the Hohmann and bi-elliptic transfers will be carried out in each range for the case $r_{i}>r_{2}>r_{1}$.

1) The range $1<R<9$

For all $\mathrm{R}$ in the range $1<R<9$ and for all $S>R$, the Hohmann transfer is better than the bi-elliptic transfer as depicted in Figure 7.

2) The range $9 \leq R \leq R_{I}$

For all $\mathrm{R}$ in the range $9 \leq R \leq R_{I}$ and for all $S>R$ the Hohmann transfer is better than the bi-elliptic transfer as depicted in Figure 8 .

3) The range $R_{I}<R \leq R_{M}$

For all $R$ in the range $R_{I}<R \leq R_{M}$ and for all $S>R$ the Hohmann transfer is better than the bi-elliptic transfer as depicted in Figure 9.

4) The range $R>R_{M}$

For all $R$ in the range $R>R_{M}$ and for all $S>R$ the Hohmann transfer is better than bi-elliptic transfer as depicted in Figure 10.

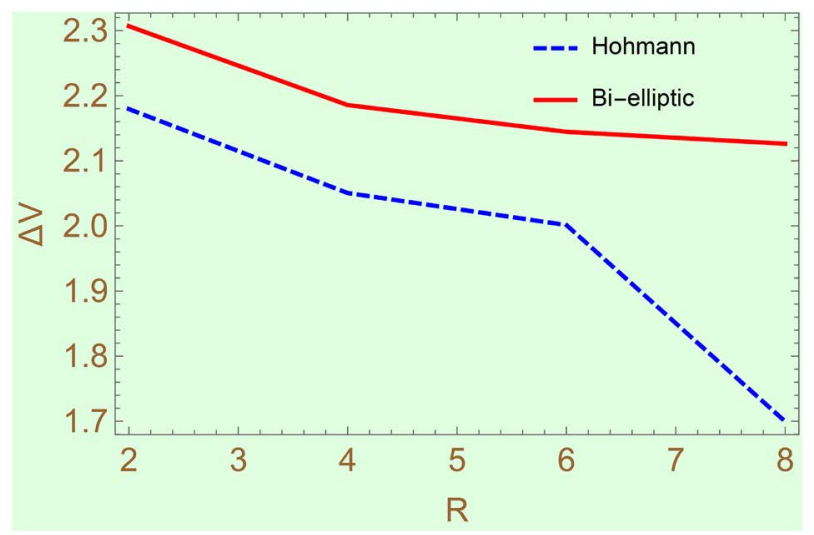

Figure 7. Graphical illustrations for $S=10$.

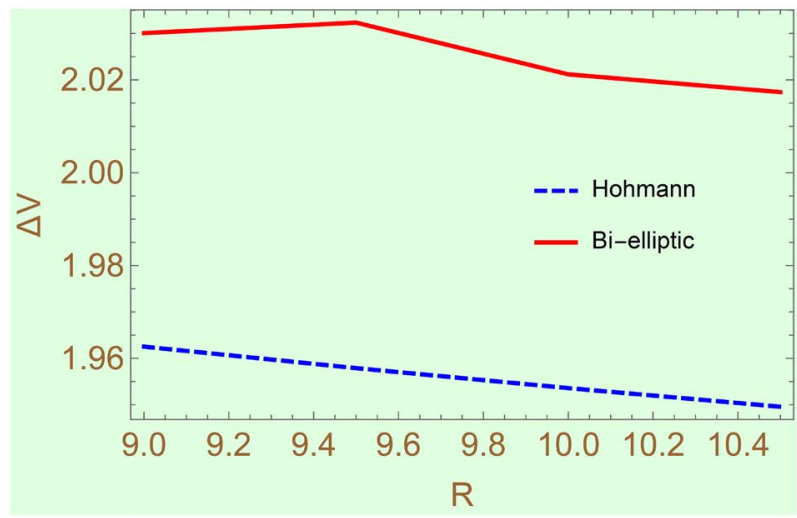

Figure 8. Graphical illustrations for $S=30$. 


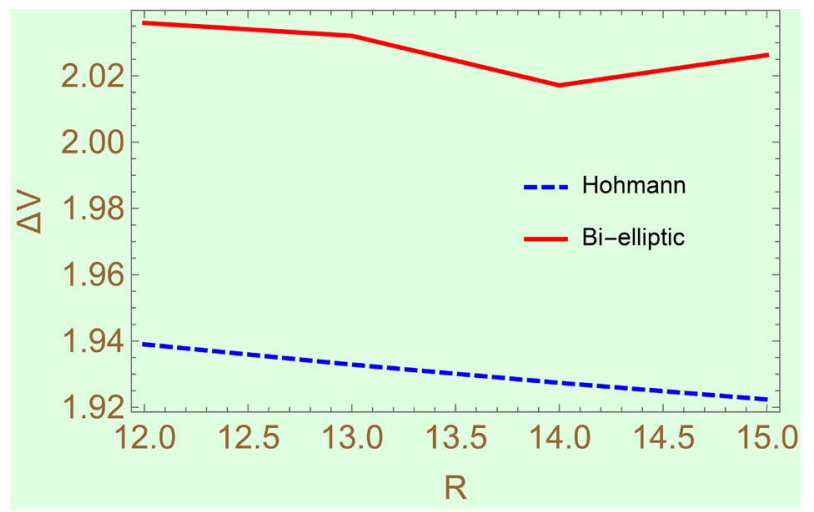

Figure 9. Graphical illustrations for $S=20$.

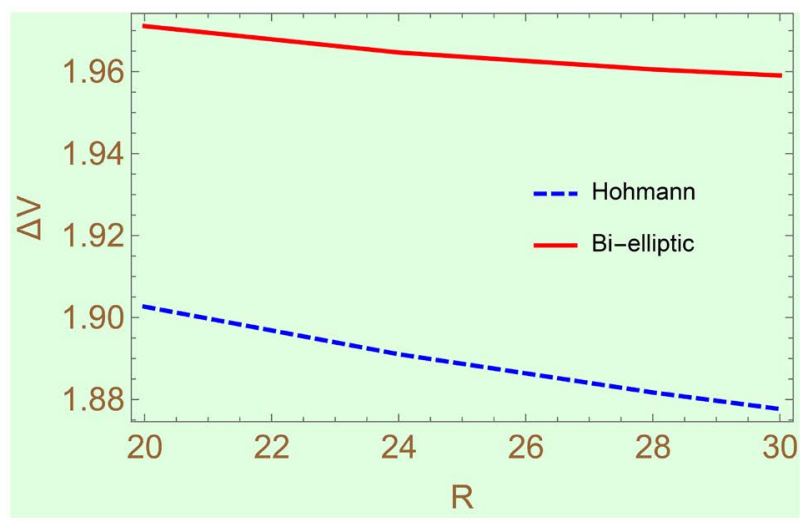

Figure 10. Graphical illustrations for $S=35$.

\section{Conclusion}

In the present paper, certain mathematical algorithms to investigate the two important non-coplanar tangential transfers between circular orbits called the Hohmann and bi-elliptic transfers have been devised. The obtained results from the two transfers were subjected to a comparative study in order to determine which among the two is better, and thereafter depicted the obtained results graphically. The suitable transfer was stated in each category of ranges according to the ratio between the final to the initial radii and thus became obvious to determine the best and or efficient transfer among the two. In addition, the Hohmann transfer turned out to be the best maneuvering transfer based on the demonstrated optimality and sensitivity analysis. While for computational developments, mathematical software has been utilized to simulate the obtained analytical procedures.

\section{Acknowledgements}

To the person who always supports, encourages and motivates me, to my father.

To the person by whom I saw the way of my life, to the kind heart and the tender soul, to that whose prayers surrounded me, pleased and embraced me, to my shelter when I am in trouble, to my wonderful mother. 


\section{Conflicts of Interest}

The author declares no conflicts of interest regarding the publication of this paper.

\section{References}

[1] Goddard, R.H. (1919) A Method of Reaching Extreme Altitudes. Smithsonian Inst Publ Misc Collect. https://doi.org/10.5479/sil.918318.39088014683783

[2] Hoelker, R.F. and Silber, R. (1959) The Bi-Elliptic Transfer between Circular Co-Planar Orbits, Alabama. Army Ballistic Missile Agency, Redstone Arsenal, (DA Tech Memo 2-59).

[3] Hohmann, W. (1960) The Attainability of Heavenly Bodies. NASA, Washington.

[4] Walter, U. (2012) Astronautics: The Physics of Space Flight. Wiley-VCH Verlag $\mathrm{GmbH} \& \mathrm{Co}$ KGA.

[5] Chobotov, V. (2002) Orbital Mechanics, Reston, Virginia. American Institute of Aeronautics and Astronautics, Inc., USA.

[6] Vallado, D.A. (2007) Fundamentals of Astrodynamics and Applications. 3rd Edition, Springer, Berling.

[7] Curtis, H.D. (2014) Orbital Mechanics for Engineering Students. 3rd Edition, OXF, UK. https://doi.org/10.1016/B978-0-08-097747-8.00006-2

[8] Mayada, J.H., Wafaa, A.Z. and Ahmed, K.I. (2015) Coplanar Maneuvers Transfer for Mission Design with Lowest $\Delta v$ Using Series Solution.

[9] Kiriliuk, E.V. and Zaborsky, S.A. (2017) Optimal Bi-Elliptic Transfer between Two Generic Coplanar Elliptical Orbits. Acta Astronautica, 139, 321-324.

https://doi.org/10.1016/j.actaastro.2017.07.006 